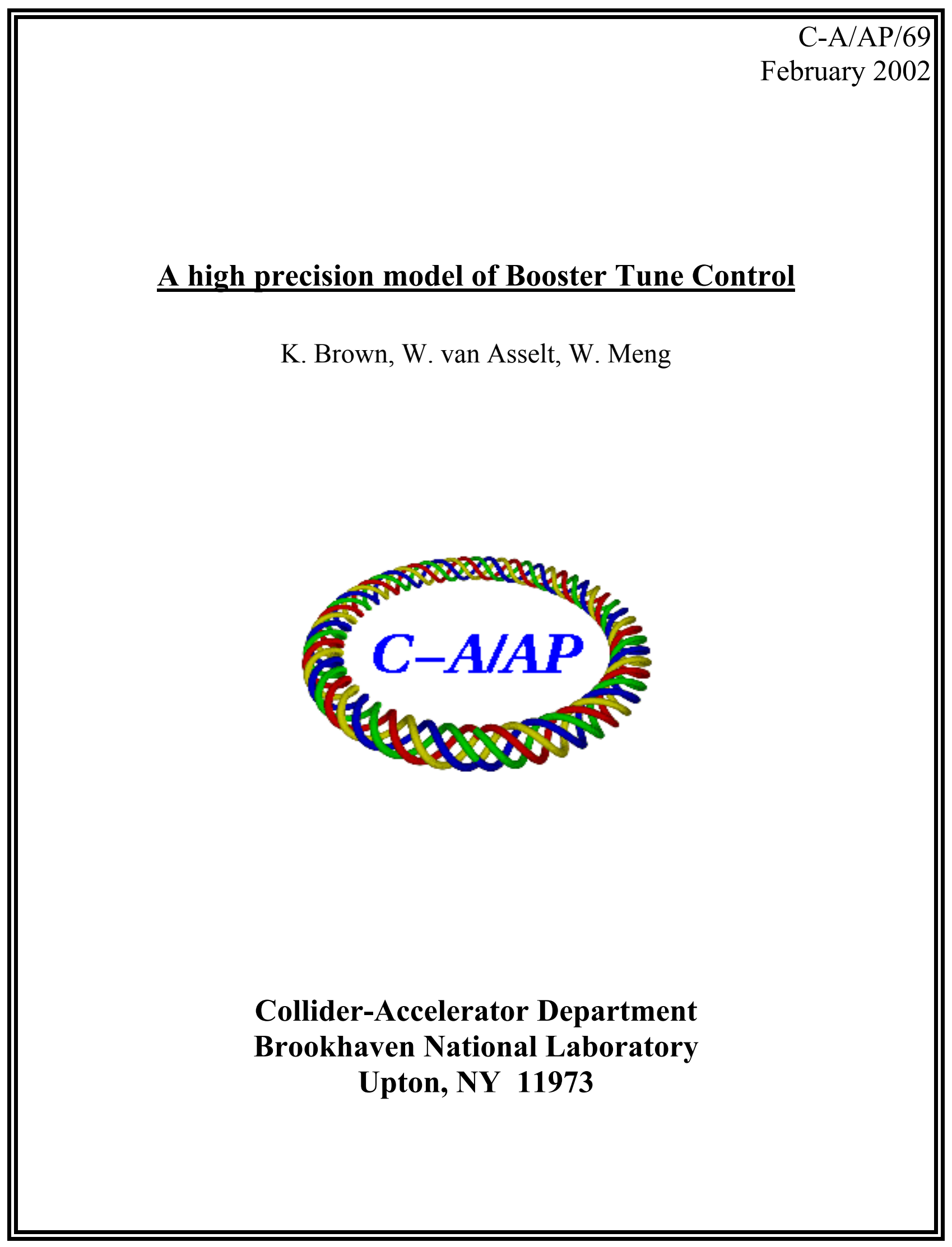


Accelerator Division

Collider Accelerator Department

Brookhaven National Laboratory

Upton, New York 11973

\title{
A high precision model of Booster Tune Control
}

\author{
Kevin Brown, Willem van Asselt, Wuzheng Meng
}

February 12, 2002

\begin{abstract}
In this note we will describe the process of developing a high precision model of the Booster betatron tunes. The Booster is used as the pre-injector to the AGS for heavy ion, polarized proton, and high intensity proton operations. In addition, the Booster is currently being modified to supply slow extracted beams to the Booster Applications Facility (BAF) [3]. The Booster is capable of accelerating these various beams up to a maximum rigidity of $17 \mathrm{~T}-\mathrm{m}$. However, for AGS injection, the Booster never operates higher than about 10 T-m [1]. For BAF operations, the Booster will operate up to the highest rigidity for heavy ion beams and up to $13 \mathrm{~T}-\mathrm{m}$ for proton beams [3]. In this note we will give a full description of the Booster tune quadrupoles and the arc dipoles. We will describe magnetic measurements and actual bare tune measurements. We will describe a 3 dimensional model we developed in order to understand various aspects of the tune quadrupole magnets that were or could not be measured directly. We will present data on tune shifts caused by $\dot{B}$ effects (e.g., vacuum chamber eddy currents) and results of a 3 dimensional model of eddy currents. Finally we will describe a high precision MAD model of the Booster tunes and the predicted tune control ranges at the highest Booster rigidities.
\end{abstract}




\section{Contents}

1 Introduction 3

2 Description of the Booster Tune Quadrupoles 4

3 Magnetic Measurements $\quad 6$

4 Booster Tune Measurements $\quad 7$

$5 \quad 3$ Dimensional Model of the Tune Quadrupoles 9

6 Modeling Booster Tunes in MAD 10

7 Vacuum Chamber Eddy Current and $\dot{B}$ Effects 13

8 Eddy Current Effects of Eared Vacuum chambers 16

9 Booster Tune Control at High Fields $\quad 17$

10 Conclusions $\quad 18$

$\begin{array}{ll}\text { A Polynomial Coefficients } & 19\end{array}$

\section{List of Tables}

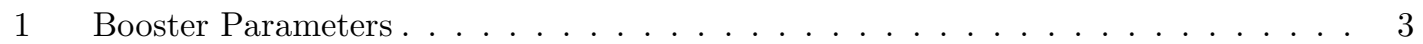

2 Booster Main Quadrupoles Parameters . . . . . . . . . . . . . . . . . 5

3 Parameters used by Booster lattice file . . . . . . . . . . . . . . . . . . . . 10

4 Polynomial coefficients .......................... 19

\section{List of Figures}

1 Opera3D model of a booster long quadrupole . . . . . . . . . . . . . . . . . 4

2 DQ3, DQ5, and FQ5 Vacuum chambers cross section . . . . . . . . . . . . 5

3 Measured normalized gradient in long and short quadrupoles . . . . . . . . . . . 6

4 bare machine tune measurements and prediction . . . . . . . . . . . . . . . 7

5 bare machine tune measurements vs $\frac{\dot{B}}{B}$. . . . . . . . . . . . . . . . . 8

6 Tune quadrupole effective lengths as a function of current . . . . . . . . . . . . . . 9

7 bare tunes prediction with measured data . . . . . . . . . . . . . . . . 12

8 Opera3D model of a booster long quadrupole, showing vacuum chamber eddy currents. In the right hand figure the iron core of the magnet was taken out to show the vacuum chamber more clearly. . . . . . . . . . . . . . . . 13

9 bare tunes prediction using 3D transient model . . . . . . . . . . . . . . . . . . . 14

10 bare tunes prediction using 3D transient model and power supply response to $\dot{B}$. 16

11 Tune control at high field, using \pm 1000 amp in tune trim supplies. . . . . . . . . . 17

12 bare tunes per the new MAD model and per OpticsControl program . . . . . . . . 18 


\section{Introduction}

Previous studies of the range of tunes that the Booster could reach when at high rigidities did not take into account all the saturation affects that occur in the main Booster dipoles and quadrupoles [2]. One reason for this is that the magnetic measurements of the Booster quadrupoles did not include effective length measurements, but just the integrated gradient [4] [5]. Since MAD expects a length and a normalized gradient $\left(K_{1}=\frac{1}{B \rho} \frac{\partial B_{r}}{\partial r}\right)$ as parameters for a quadrupole, using a fixed length and a gradient based on integrated field measurements will not predict the tunes accurately. This is because the integrated field has to be re-expressed to be interpreted as a gradient in the center of the quadrupole. I.E.,

$$
\left.\frac{\partial B_{r}}{\partial r}\right|_{z=0}=\frac{1}{L_{e f f}} \int_{-\infty}^{\infty} \frac{\partial B_{r}}{\partial r} d l
$$

Since $L_{e f f}$ is not a constant, but varies as a function of field at the pole tips, the value used by MAD must vary as the field increases.

In this report we take a slightly different approach to achieving a higher precision prediction of the Booster tunes. We have used measured bare tunes data to help guide us in developing a better model based on magnetic measurements and a 3 dimensional model of the Booster quadrupoles. This new model uses a fifth order polynomial to generate a normalized gradient based on the integrated gradient measurements,

$$
K_{1}=\frac{1}{B \rho L_{e f f}}\left(a_{0}+a_{1} \cdot I_{q}+a_{2} \cdot I_{q}^{2}+a_{3} \cdot I_{q}^{3}+a_{4} \cdot I_{q}^{4}+a_{5} \cdot I_{q}^{5}\right)
$$

where the coefficients, $a_{0}, a_{1} \ldots$ are derived by performing a least squares linear regression fitting to the measured magnetic data. $L_{\text {eff }}$ is derived from a third order polynomial of length as a function of quadrupole current, based on the 3 dimensional model of the quadrupoles. $B$ is derived from an eighth order polynomial mapping the measured fields of the arc dipoles as a function of current. In addition the model uses an eighth order polynomial to map the measured gradient of the arc dipoles as a function of current in the arc dipoles [7]. An accurate $B$ is required to allow the proper normalization of the quadrupole gradients based on our best knowledge of $B \rho$ of the Booster. Finally, we have included in our model the change in gradient caused by the affects resulting from the $\dot{B}$, such as eddy currents in the tune quadrupole vacuum chambers, by including a parameter for the $\dot{B}$ of the Booster ramp [section 7]. For details on the polynomials that we used and the coefficients that were derived see appendix A.

Table 1: Booster Parameters

\begin{tabular}{l|c}
\hline Parameter & Value \\
\hline Circumference & $201.78(1 / 4 \mathrm{AGS}) \mathrm{m}$ \\
Ave. Radius & $32.114 \mathrm{~m}$ \\
Magnetic Bend R & $13.86557 \mathrm{~m}$ \\
Lattice Type & Separated Function, FODO \\
No. Superperiods & 6 \\
No. of Cells & 24 \\
Betatron Tunes,X,Y & $4.82,4.83$ \\
Vacuum Chamber & $70 \times 152 \mathrm{~mm}$ Dipoles \& $152 \mathrm{~mm}($ circular $)$ Quads \\
Max. Rigidity & $17 \mathrm{Tm}$ \\
Injection Rigidity & $2.2 \mathrm{Tm}(200 \mathrm{MeV}$ protons $) \& 0.9 \mathrm{Tm}(1 \mathrm{MeV} / \mathrm{nuc} \mathrm{Au}(32+))$ \\
Acceleration Rate & $8.9 \mathrm{~T} / \mathrm{s} \mathrm{up} \mathrm{to} 8 \mathrm{Tm}(7.5 \mathrm{~Hz}) \& 1 \mathrm{~T} / \mathrm{s} \mathrm{up} \mathrm{to} 17 \mathrm{Tm}(0.7 \mathrm{~Hz})$ \\
\hline
\end{tabular}




\section{Description of the Booster Tune Quadrupoles}

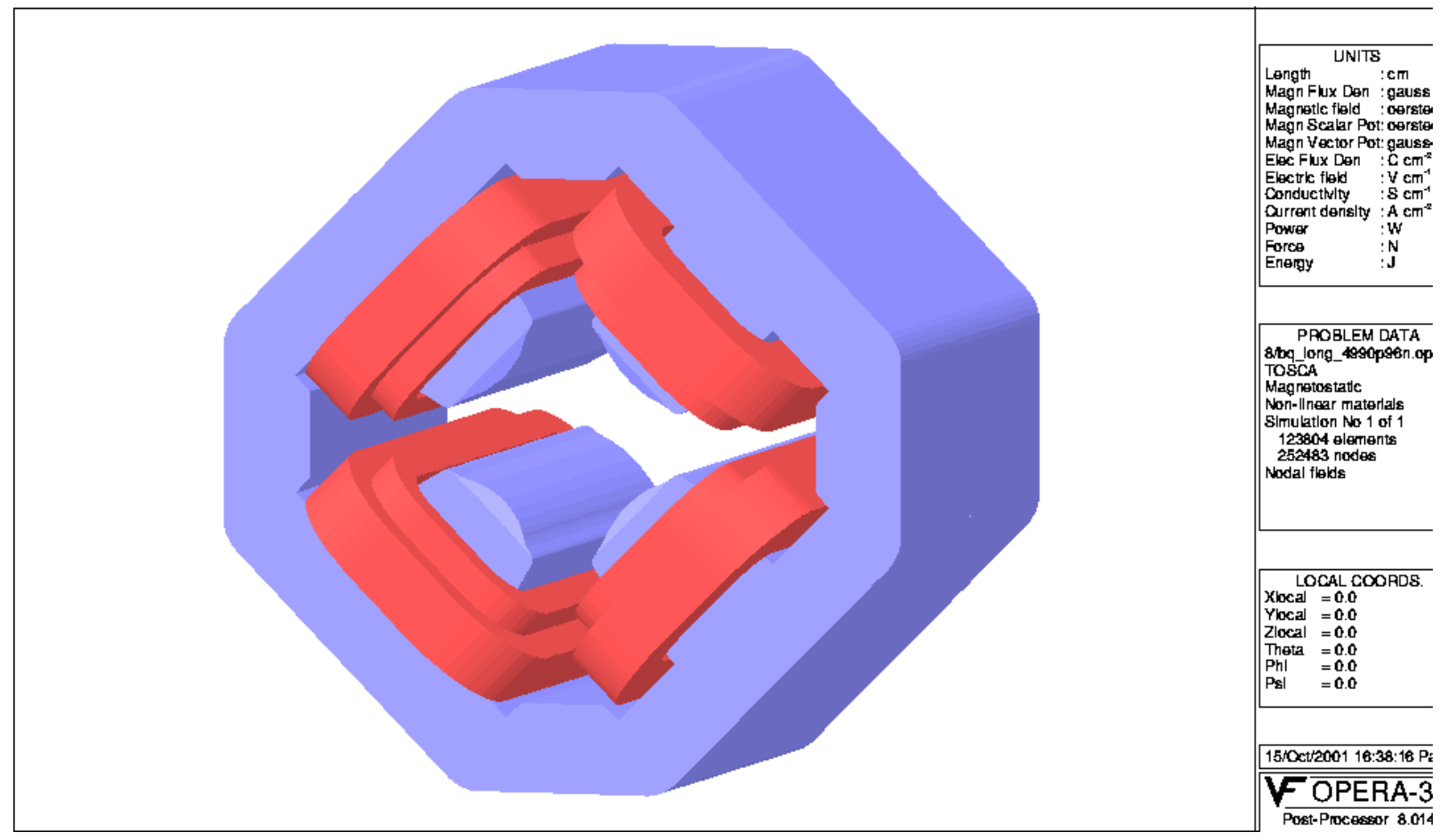

Figure 1: Opera3D model of a booster long quadrupole

The Booster lattice is built as a separated function, FODO type lattice in which the defocusing quadrupoles are slightly longer than the focusing quadrupoles. The Booster arc dipoles and the two types of quadrupoles are powered in series. Since the arc dipoles have an intrinsic focusing component, making the defocusing quadrupoles slightly longer brought the vertical tune up closer to the horizontal tune. Using the tune trim coils the tuning range at low rigidities allows shifting the vertical tune up high enough to compensate for space charge tune shifts and avoid the strong integer stop band at $\nu_{y}=4$. Stop band corrections are used to correct for all the significant resonances between $\nu_{y}=4$ and $\nu_{y}=5$.

The design bare Booster tunes were around 4.82. After most of the quadrupole cores had already been built, though, it was found, from magnetic measurements, that the strengths of the quadrupoles relative to the arc dipoles was $4.0 \%$ too low [9]. As a result, the bare Booster tunes are now around 4.63 horizontal and 4.61 vertical, at low rigidities. Table 2 gives a summary of the characteristics of the two types of quadrupoles. A complete listing of these characteristics can be found in references [4] [5].

Although the cores of the two types of magnets are different lengths, the coils are all the same length. This means the overall length of the magnets is the same, although the magnetic lengths are different. This does not significantly affect the magnetic characteristics.

Not all quadrupole vacuum chambers are round. Currently in DQ5 and FQ5 there are special "eared" chambers, as shown in figure 2. After completion of the modifications for BAF, there will be 3 such chambers in the Booster (DQ3, DQ5, FQ5). Note that all vertical quadrupoles are located at odd locations (DQ3,DQ5, etc.) and all horizontal quadrupoles are located at even locations (DQ2,DQ4, etc.). 
Table 2: Booster Main Quadrupoles Parameters

\begin{tabular}{|l|c|c|}
\hline Parameter & Short Quad & Long Quad \\
\hline Core Length & $42.55 \mathrm{~cm}(16.75 \mathrm{inch})$ & $43.82 \mathrm{~cm}(17.25 \mathrm{inch})$ \\
Number of Magnets & $24+1$ & $24+1$ \\
Radius at Pole Tip & $8.255 \mathrm{~cm}(3.25 \mathrm{inch})$ & $8.255 \mathrm{~cm}(3.25 \mathrm{inch})$ \\
Laminations & 670 & 690 \\
Thickness & $0.025 \mathrm{inch}$ & 0.025 inch \\
Vacuum Chamber & $152 \mathrm{~mm}($ circular,inconel $)$ & $152 \mathrm{~mm}$ (circular,inconel) \\
Main Coil Turns & 5 (water cooled) & 5 (water cooled) \\
Main Coil Max. I & 5000 amp & 5000 amp \\
Tune Coil Turns & 1 & 1 \\
Tune Coil Max. I & $1100 \mathrm{amp}$ & $1100 \mathrm{amp}$ \\
\hline
\end{tabular}

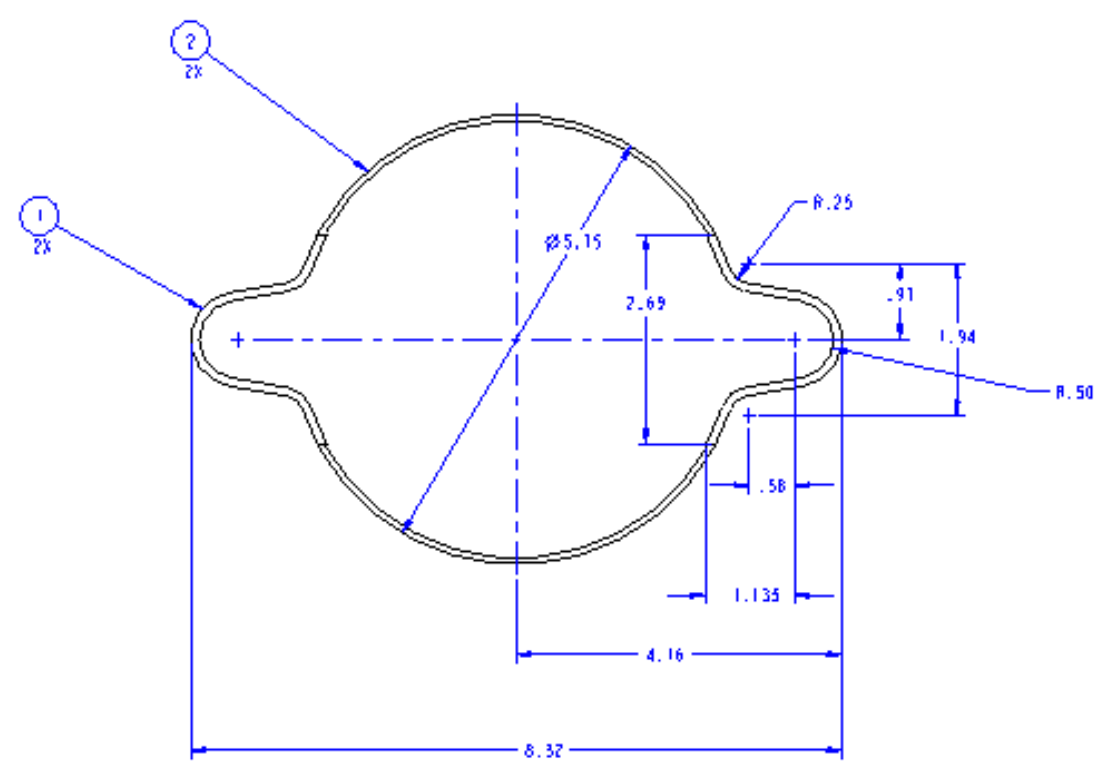

Figure 2: DQ3, DQ5, and FQ5 Vacuum chambers cross section 


\section{Magnetic Measurements}

References [4] [5] [7] describe in full detail the magnet measurements of the Booster quadrupoles and main arc dipoles. Figure 3 shows the normalized gradient of the booster long and short quadrupoles as a function of current.

According to references [4] [5] the specified accuracy in the RMS spread of the fractional variation in integrated field was $0.1 \%$. The magnets in the measurement sample all agreed to within $0.05 \%$ (standard deviation), for both long and short quadrupoles. Unfortunately a detailed error analysis was not given, leaving open many questions about different sources of error and systematics. A more detailed analysis of the short quadrupole production measurements did appear in a later technical note [6], but no such analysis was published for long quadrupole production measurements. For the short magnets, anomalies were noted (for two of the 25 magnets produced) but no cause was ever found. Two of the magnets deviated by 0.4 and $0.5 \%$ from the average.

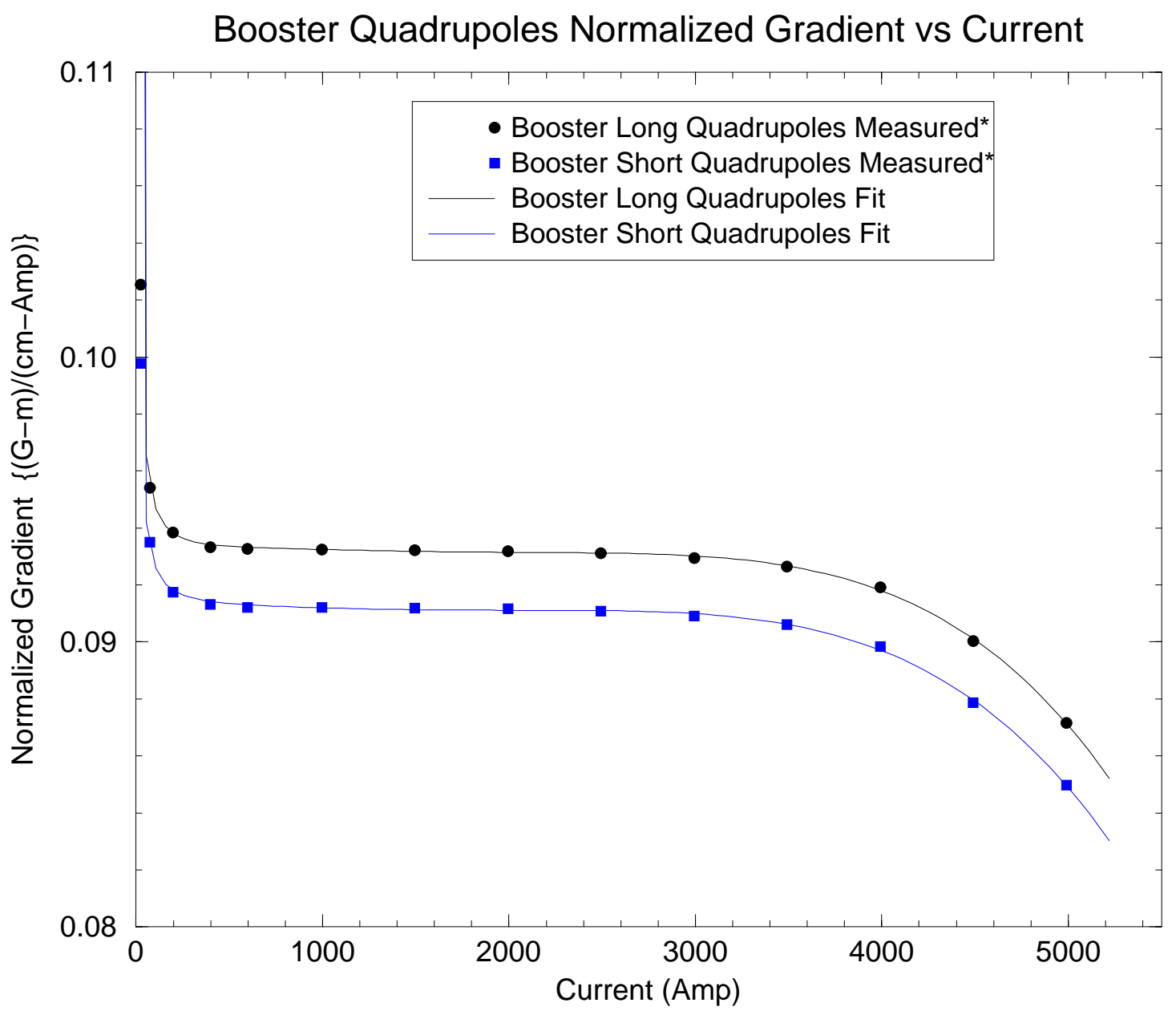

* Booster Technical Note No. 174/176, E. Bleser 1990

Figure 3: Measured normalized gradient in long and short quadrupoles 


\section{Booster Tune Measurements}

In the 1992 and 1993 Booster commissioning notebooks we found two independent sets of Booster bare machine tune measurements. For these measurements the same procedure was followed. In each case the tune quadrupole power supplies were left on, but with zero current (this was to prevent back-emf from the main windings from pulling field out of the magnets), the chromaticity functions were set for zero chromaticity (non-zero currents in sextupoles), the beam intensity was made low (to avoid space charge tune shifts at injection), and the RF was left on (radius set to zero) during the main magnet invert (to allow measurements during negative B-dot). What is uncertain in these measurements is what the chromaticity really was, how close the radius was at, and stayed at, the center of the quadrupoles, and whether the orbit was corrected or not. In addition the data did not include the current error in the tune quadrupoles (how much the real current deviated from zero as a function of time or $\dot{B}$ ). In any case the measured bare tunes for the two sets are very consistent with each other. Unfortunately they do not agree with the canonical MAD prediction of the static bare tunes. In addition, these measurements show a large affect from the B-dot. Figure 4 shows the data. Figure 5 shows the tune shifts relative to a zero $\dot{B}$ tune as a function of $\frac{\dot{B}}{B}$.

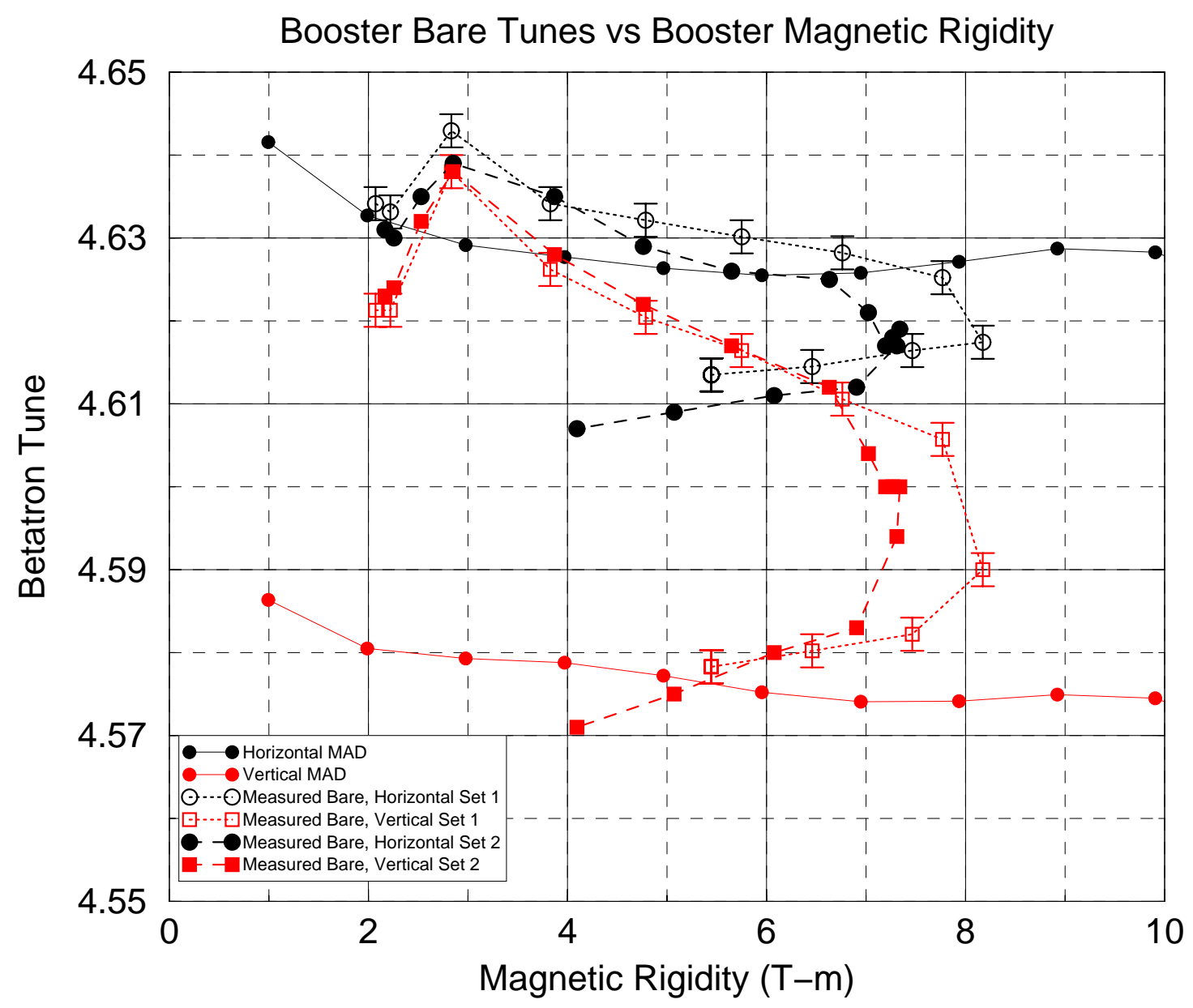

Figure 4: bare machine tune measurements and prediction 


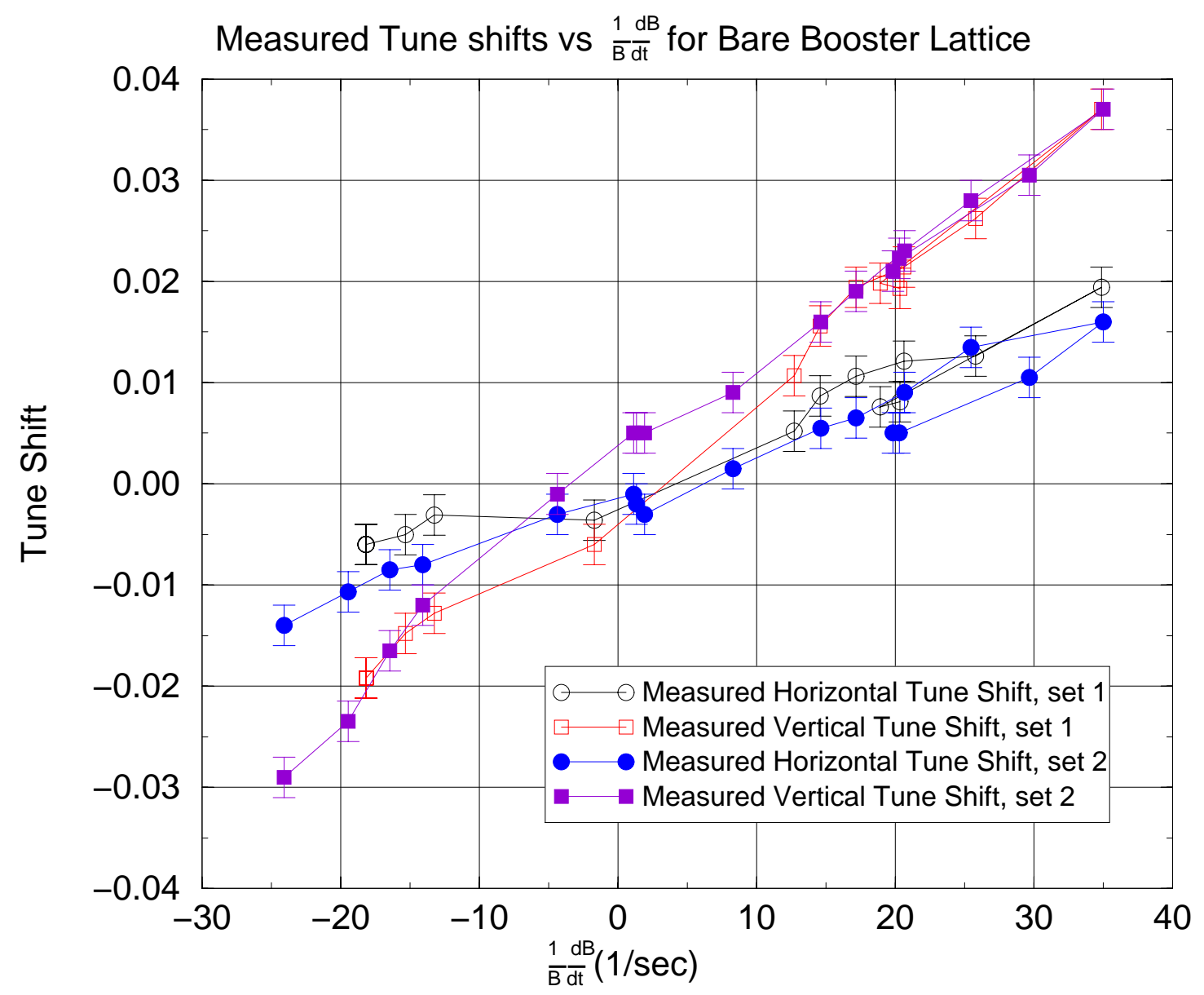

Figure 5: bare machine tune measurements vs $\frac{\dot{B}}{B}$. 


\section{Dimensional Model of the Tune Quadrupoles}

Since we did not have measurements of the effective length of the quadrupoles as a function of current (or field) we developed a 3 dimensional model of both the short and long quadrupoles using Opera3D. An image of the long quadrupole for this model is shown above, in figure 1. The results of the the modeled effective lengths are shown in figure 6 . This data was fitted to a third order polynomial, which is used in the MAD lattice file to define the length of the Booster quadrupoles. The predicted bare tunes shown in figure 4 were calculated using this polynomial, along with the measured field gradients in both the quadrupoles and the arc dipoles.

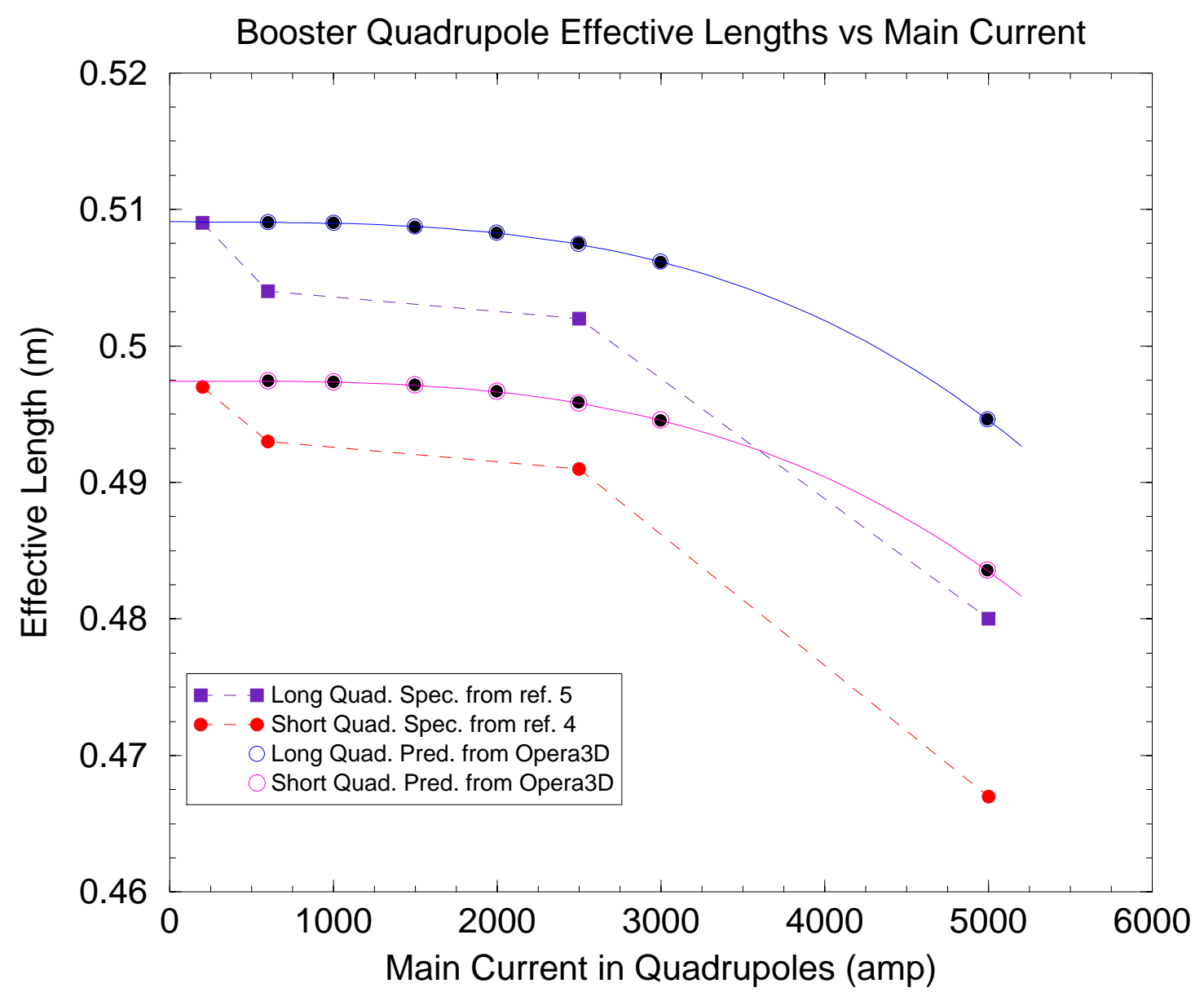

Figure 6: Tune quadrupole effective lengths as a function of current 


\section{Modeling Booster Tunes in MAD}

We have already given rough descriptions of the MAD lattice that was used to model the Booster. This section will give a more detailed explanation of this lattice, and how to make use of it. The lattice is publicly available in the / rap/lattices/agsbooster directory on owl.pbn.bnl.gov and the Sun workstations. Questions about these files can always be addressed to the authors. These lattice files were built for bnlmad, which has slightly different syntax from the CERN Mad8, and so it is not guaranteed to work with Mad8 or Mad9.

The Booster lattice file has evolved from original lattices developed during the design and construction of the Booster. Various people have contributed to it, adding further and further sophistication to the model. This has also made reading the lattice file (which describes a "flat" lattice) rather difficult. Whenever possible we have added comments to the existing file. Table 3 gives a short list of user defined parameters and what they do. Examples of values to use for these are given in the BOOSTER.PWR file.

Table 3: Parameters used by Booster lattice file

\begin{tabular}{l|l}
\hline Parameter & meaning \\
\hline IDIPO & main dipole current, in amp \\
BRHO & Calculated using BDIPO and RHO (Larc/bend angle) \\
IQHC & horizontal tune trim quadrupole current, in amp \\
IQVC & vertical tune trim quadrupole current, in amp \\
ISH & horizontal chrom. sextupole current, in amp \\
ISV & vertical chrom. sextupole current, in amp \\
ISEBC8F8 & dynamic sextupole current, for slow extraction \\
ISEBB4E4 & dynamic sextupole current, for slow extraction \\
BDOT & B-dot variable $(\mathrm{T} / \mathrm{s})$ \\
\hline
\end{tabular}

The main dipole current (IDIPO) is used as the variable in polynomial expansions, as the main current in the tune quadrupoles, and to calculate the normalization factor $B \rho$. For example,

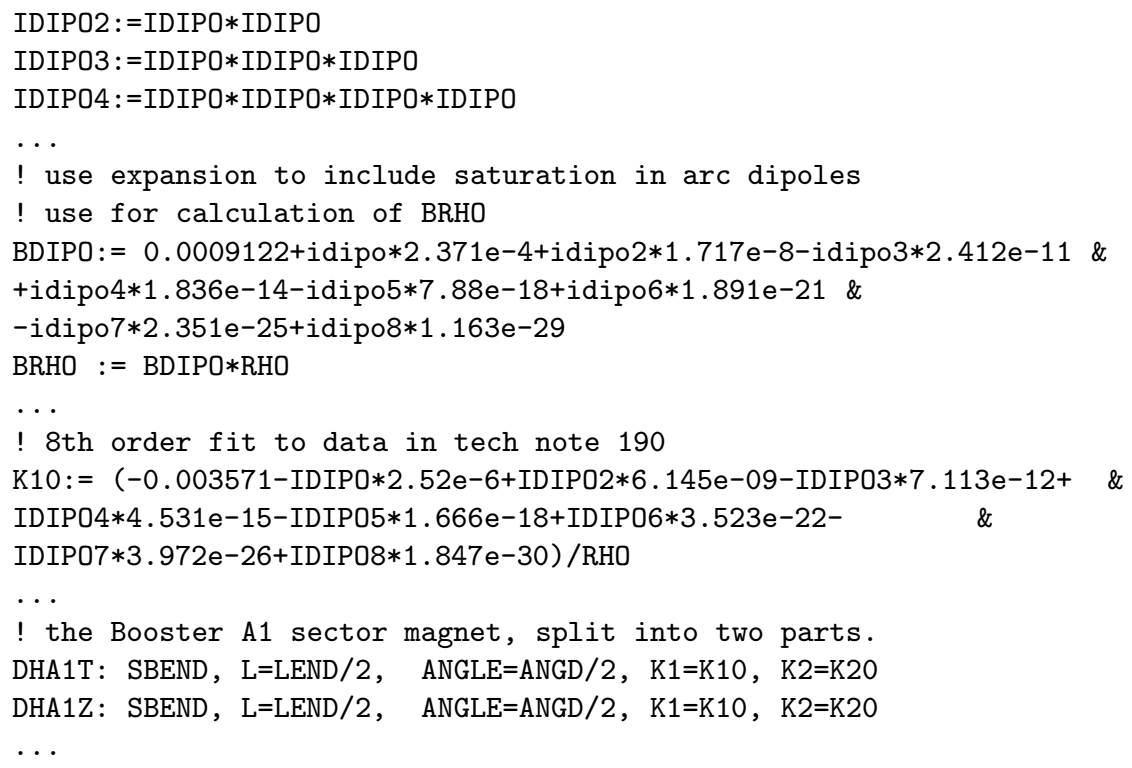

To calculate the tune quadrupole coefficients we do the following:

! To match the change in power supply response to back emf due to bdot we add 


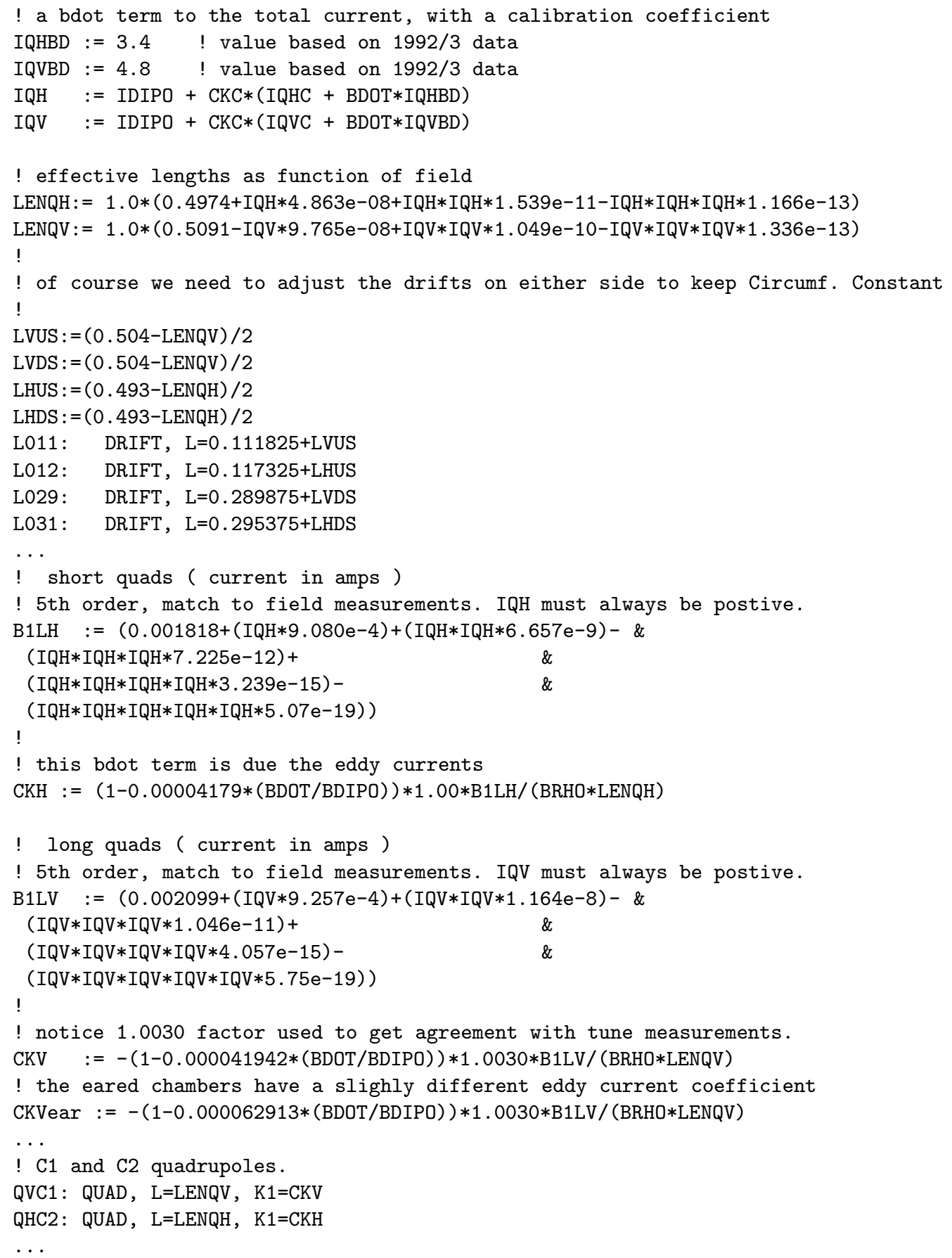

After considering all we knew about the quadrupoles, from magnetic measurements and from a static 3D model, we still end up with a discrepancy between the measured and predicted bare tunes. We went through various modeling exercises to try and see what would explain the difference. Altering the quadrupole coefficients of the main dipoles does not explain the effect, since this causes both the horizontal and vertical tunes to shift more or less equally. Altering the lengths of the quadrupoles cannot explain the difference, since the change would have to be unrealistically large. Finally we found that by just increasing the strength of the long quadrupoles by $0.3 \%$ brought the prediction into perfect agreement with the measurements. Figure 7 shows the model with this $0.3 \%$ adjustment included.

In an effort to explain the $0.3 \%$ difference we went back to the $3 \mathrm{D}$ magnet model. In [4] 
and $[5]$ it is noted that for both the long and the short magnets the same measurement coil was used. The measurement coil was 36.5 inches long. We modeled the two types of magnets and integrated the gradient over 36.5 inches and over 72 inches and compared the two cases. In each case the discrepancy was on the order of $0.1 \%$ or less. So this argument doesn't take us far enough to offer an explanation. The same reports also tell us that the RMS distribution of the magnets in the sample set had differences of the order of $0.05 \%$. We conclude that at best we can speculate that $0.1 \%$ of the affect can be due to using the same measurement coil for both short and long magnets.

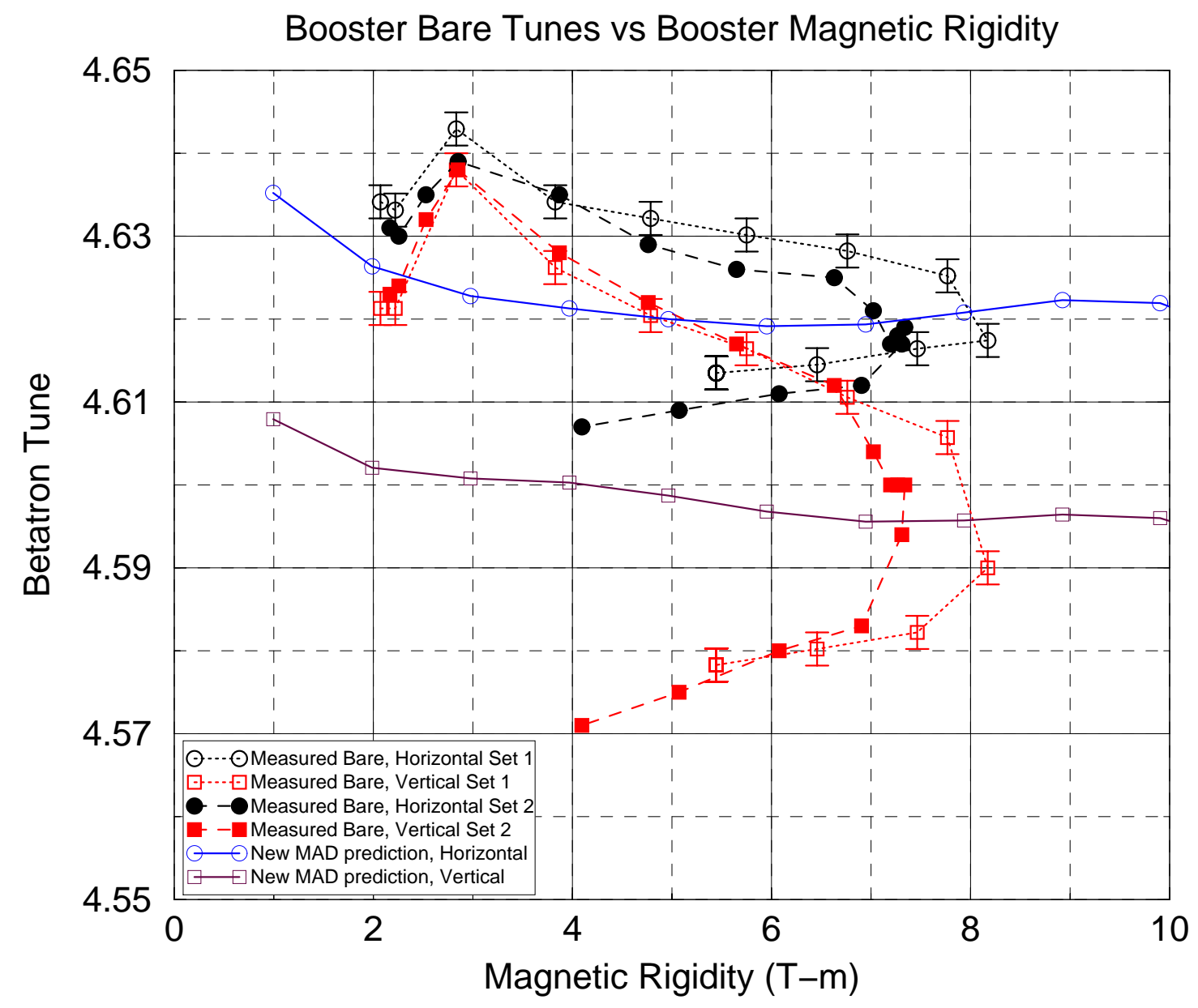

Figure 7: bare tunes prediction with measured data 




Component: JZ

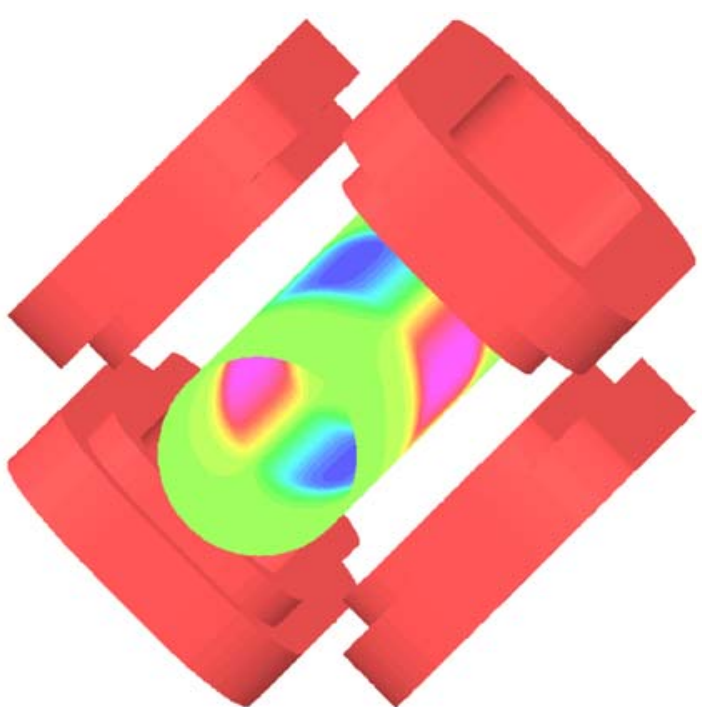

0.99191899919519

Figure 8: Opera3D model of a booster long quadrupole, showing vacuum chamber eddy currents. In the right hand figure the iron core of the magnet was taken out to show the vacuum chamber more clearly.

\section{Vacuum Chamber Eddy Current and $\dot{B}$ Effects}

To model the vacuum chamber eddy current effects we first created a 2 dimensional model of the quadrupoles with a round Inconel vacuum chamber. The purpose of this was to see whether eddy currents in the quadrupoles were significant enough to explain the measured tune shifts. Since it was a $2 \mathrm{D}$ model we could not show how the long and short quadrupoles were really affected, and the predicted tunes shifts were equal, but significant. We next created a 3D model and found there is little difference in the two types of magnets. Figure 9 shows the predicted tunes along with the measured tunes for one of the sets of measured data. It is immediately apparent that the eddy currents do not, by themselves explain the observed $\dot{B}$ effects. In fact they cause the gradient to be reduced, decreasing the tunes, not increasing.

Figure 8 shows the Opera3D model of a long quadrupole with a vacuum chamber. The zcomponent of the current density in the pipe is shown with different colors for different current densities. Those shown in the figure correspond to the maximum $\frac{\partial B}{\partial t}$ that was modeled. The eddy currents flow inside the vacuum chamber around the pole tips, with red/pink indicating current flowing out of the paper (clockwise for the upper right coil). Current in the coils flows in the opposite direction (counterclockwise for the upper right coil). The result is a reduction in the gradient seen by the beam. 


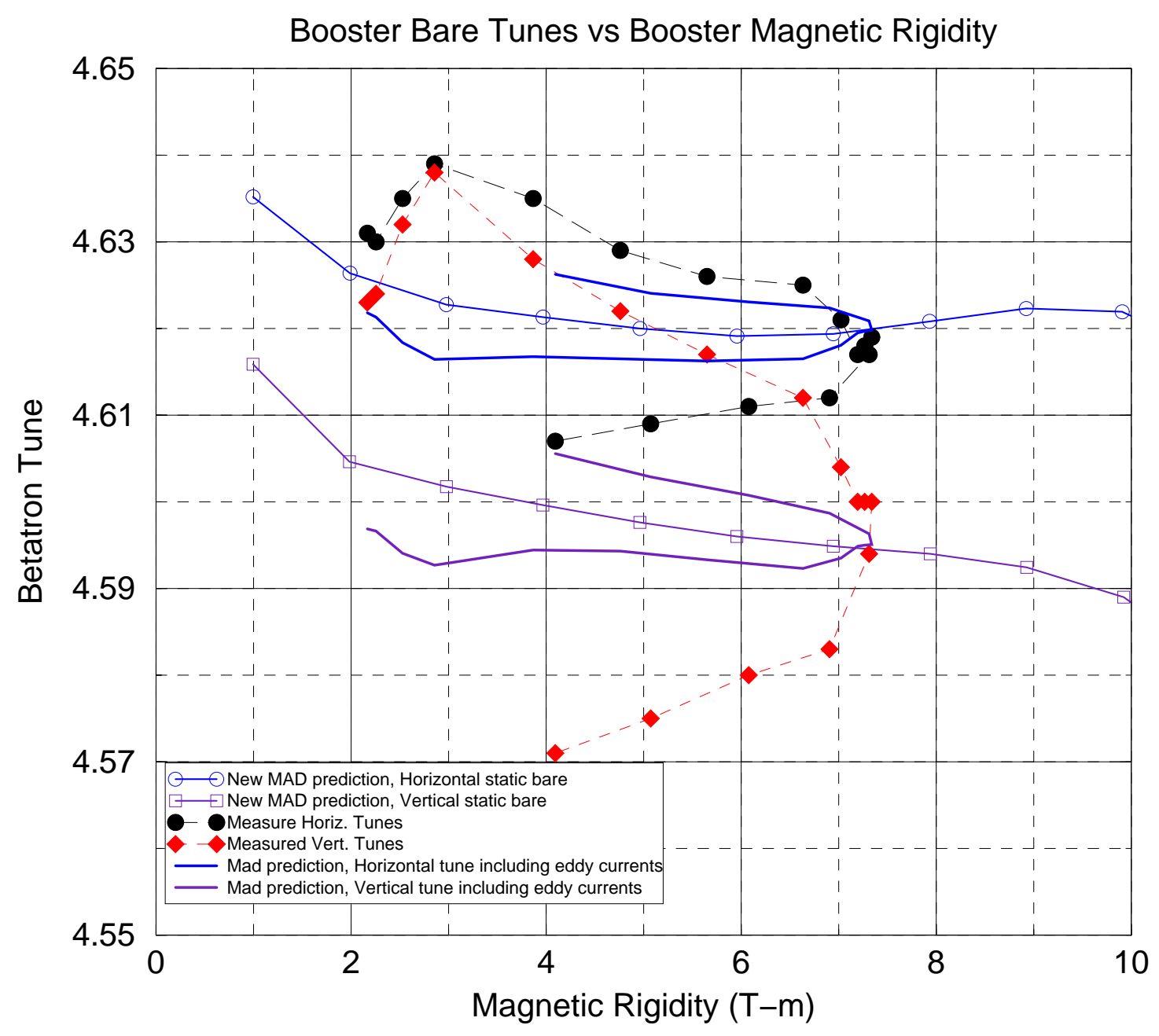

Figure 9: bare tunes prediction using 3D transient model 
The only way for the bare tunes to increase with increasing $\dot{B}$, is for the gradient in the magnets to increase with increasing $\dot{B}$. Since the data measuring the $\dot{B}$ effect does not include the measured currents in the tune quadrupole power supplies, we cannot know how large or small this current was. We can observe the currents today and observe on the order of 10 or so amps on the current read-backs during the the Booster acceleration ramp when the reference is made to be zero throughout the cycle (and the sign is correct, to increase the gradient). Incorporating this effect into the model, we added to the currents a $\dot{B}$ term, with a set of calibration coefficients, derived to match the measurements. To match the measured data the calibration coefficients need to be $C_{H}=3.4$ and $C_{V}=4.8 \mathrm{amp} / \mathrm{T} / \mathrm{sec}$, which would put about $30 \mathrm{amp}$ additional current through the magnets at maximum $\dot{B}$, which is consistent with what we measure today.

$$
\begin{aligned}
& I_{H}=I_{D I P O L E}+0.2 \cdot\left(I_{\text {Htrim }}+\dot{B} \cdot C_{H}\right) \\
& I_{V}=I_{D I P O L E}+0.2 \cdot\left(I_{\text {Vtrim }}+\dot{B} \cdot C_{V}\right)
\end{aligned}
$$

The equations used in the model are: for the short quadrupoles,

$$
K_{1}=\left(1-0.00004179 \cdot \frac{1}{B} \frac{\partial B}{\partial t}\right) \cdot \frac{1}{B \rho L_{e f f}}<\frac{\partial B_{r}}{\partial r}>
$$

and for the long quadrupoles,

$$
K_{1}=-\left(1-0.000041942 \cdot \frac{1}{B} \frac{\partial B}{\partial t}\right) \cdot \frac{1.003}{B \rho L_{e f f}}<\frac{\partial B_{r}}{\partial r}>
$$

and for the long quadrupoles with eared vacuum chambers,

$$
K_{1}=-\left(1-0.000062913 \cdot \frac{1}{B} \frac{\partial B}{\partial t}\right) \cdot \frac{1.003}{B \rho L_{e f f}}<\frac{\partial B_{r}}{\partial r}>
$$

where $L_{e f f}$ and $\left\langle\frac{\partial B_{r}}{\partial r}>\right.$ are derived from the respective polynomials (two sets, one for shorts and one for longs), and $B$ is derived from the polynomial expansion for the main arc dipoles. 


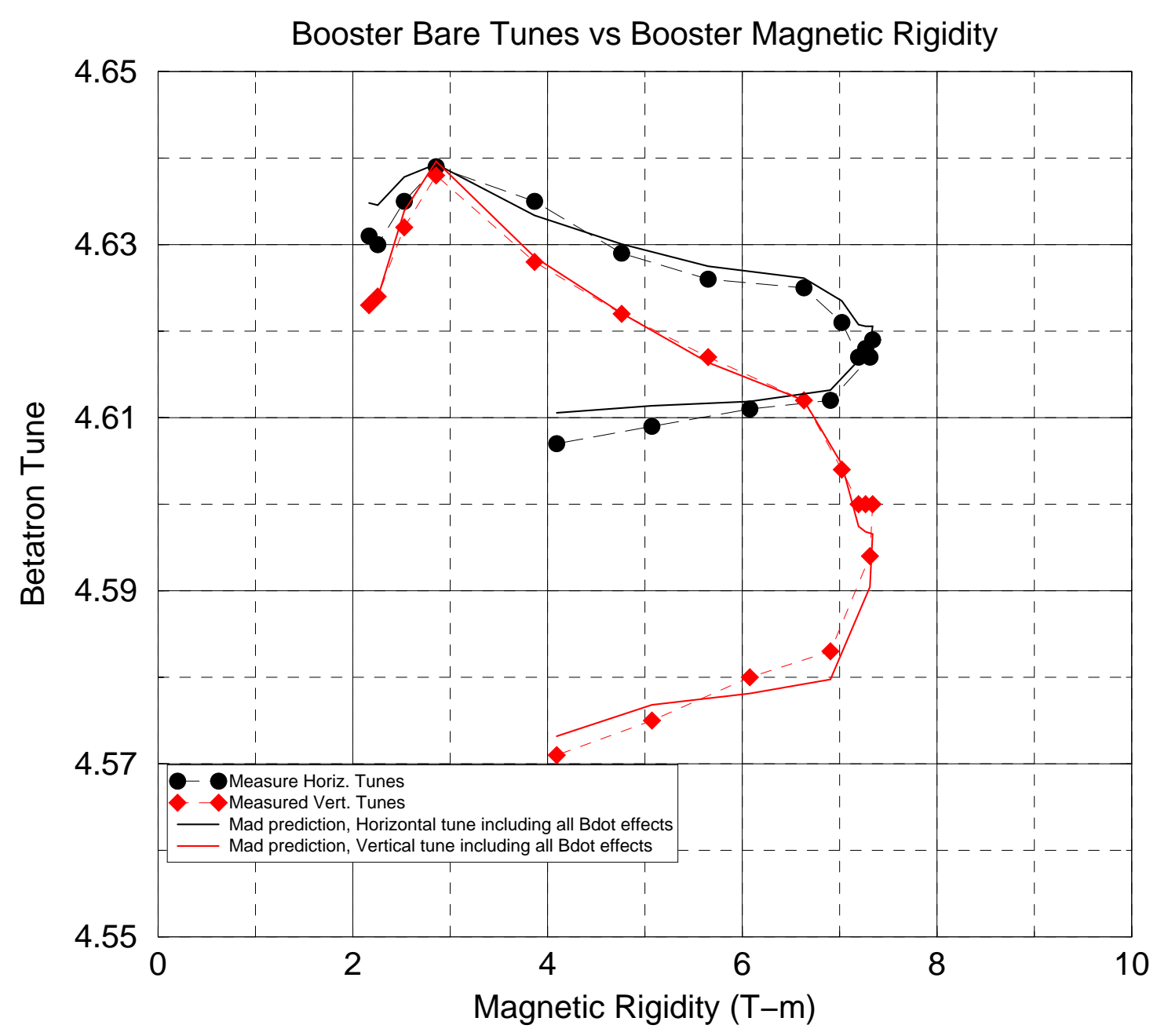

Figure 10: bare tunes prediction using 3D transient model and power supply response to $\dot{B}$

\section{Eddy Current Effects of Eared Vacuum chambers}

The eared vacuum chambers also change the quadrupole moment as a function of $\dot{B}$. Since the eared vacuum chambers are thicker than the normal vacuum chambers, the eddy currents are larger by about $25 \%$. Due to the symmetry of the structure the higher order field components for sextupole, octupole, and above tend to cancel out, and have magnitudes that are insignificant. This means the stop band strengths do not change as function of $\dot{B}$. 


\section{Booster Tune Control at High Fields}

Using this new model of the Booster we can now predict how much tune space is accessible when we operate at very high fields, assuming we can change the tune trim quadrupoles by \pm 1000 amp. Figure 11 shows the amount of tune space available for rigidities from $14 \mathrm{Tm}$ to $17 \mathrm{Tm}$.

\section{Booster Tune Control At High Field}

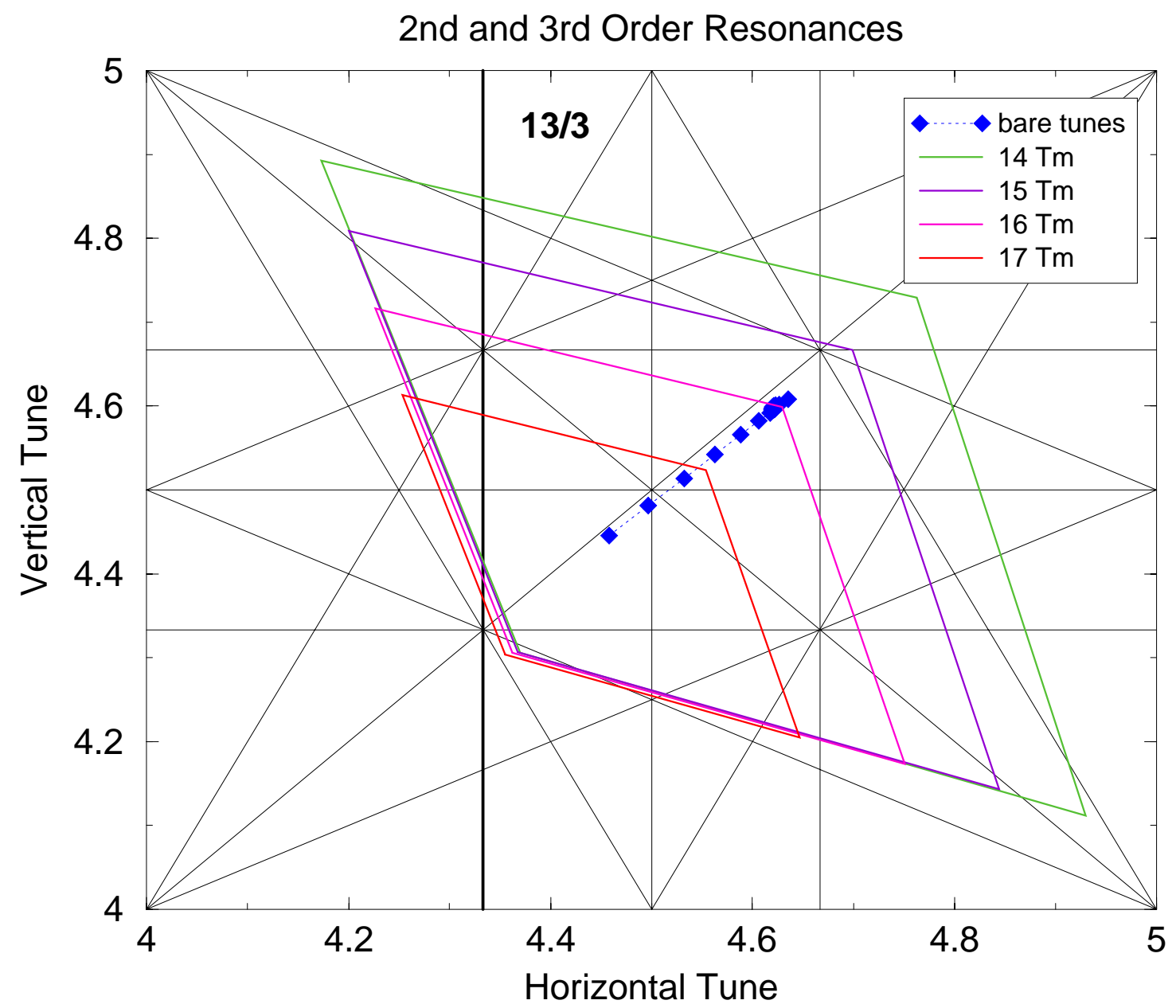

Figure 11: Tune control at high field, using $\pm 1000 \mathrm{amp}$ in tune trim supplies. 


\section{Conclusions}

We now have a precise model of the Booster tunes and tune control through the tune trim power supplies. The new model refines our knowledge incrementally from the current best model, found only in the OpticsControl program used to control the Booster tunes. Figure 12 shows our new bare tune model along with the bare tunes as derived from the OpticsControl program. In addition we have studied the affect of eddy currents in the quadrupole vacuum chambers and demonstrated that measured tune shifts as a function of $\dot{B}$ are affected, in part, by these eddy currents, but are due more significantly to power supply response to $\dot{B}$. We have had to insert a $0.3 \%$ factor in the strength of the long quadrupoles in order to get a model which agrees with measured data. We have not been successful in finding the sources of this discrepancy but we can speculate on possible causes. Using the same measurement coil for both the short and long magnets could contribute to the error, but does not fully explain it. The data from the distributions of integrated gradients for the short quadrupoles shows that most of the magnets were built very consistently. The fact that two of the 25 magnets were significantly different does not in itself suggest a source of the error, since the integrated gradients used in the MAD model are the average of all the measured magnets.

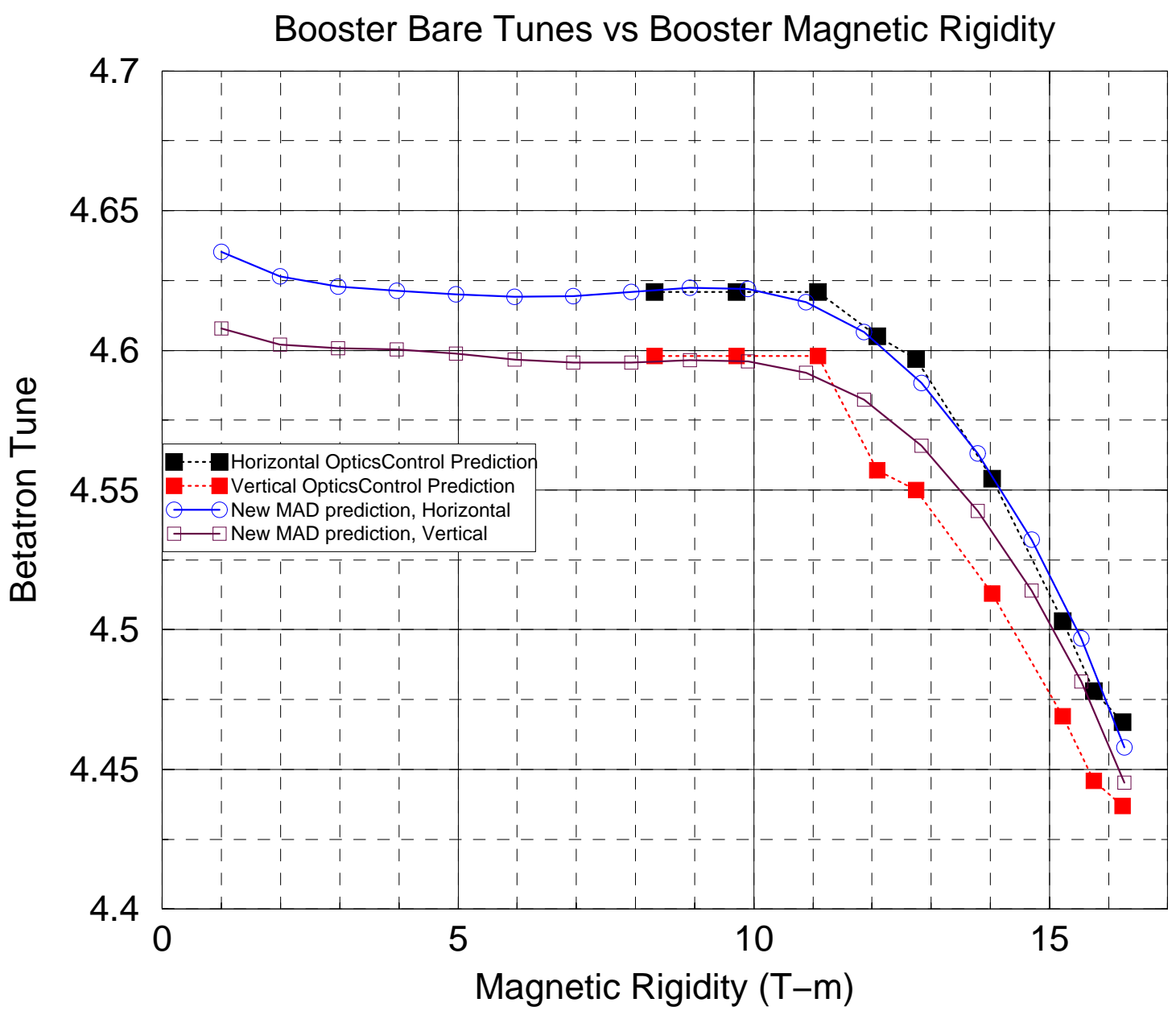

Figure 12: bare tunes per the new MAD model and per OpticsControl program 


\section{A Polynomial Coefficients}

The following table summarizes coefficients used in the various polynomials in the MAD models. Polynomials are usually used to generate a field or gradient based on current flowing through the magnet. $I_{d}$ is current in the Booster dipoles and $I_{q}$ is current in the booster quadrupoles.

$$
\begin{gathered}
B=\left(a_{0}+a_{1} \cdot I_{d}+a_{2} \cdot I_{d}^{2}+a_{3} \cdot I_{d}^{3}+a_{4} \cdot I_{d}^{4}+a_{5} \cdot I_{d}^{5}+a_{6} \cdot I_{d}^{6}+a_{7} \cdot I_{d}^{7}+a_{8} \cdot I_{d}^{8}\right) \\
K_{1}=\frac{1}{B \rho L_{e f f}}\left(a_{0}+a_{1} \cdot I_{q}+a_{2} \cdot I_{q}^{2}+a_{3} \cdot I_{q}^{3}+a_{4} \cdot I_{q}^{4}+a_{5} \cdot I_{q}^{5}\right) \\
L_{e f f}=\left(a_{0}+a_{1} \cdot I_{q}+a_{2} \cdot I_{q}^{2}+a_{3} \cdot I_{q}^{3}\right)
\end{gathered}
$$

Table 4: Polynomial coefficients

\begin{tabular}{l||l|l|l|l|l|l}
\hline Coeff. & Dipole B & Dipole K1 & Short quad K1 & Long quad K1 & Short quad Leff & Long quad Leff \\
\hline$a_{0}$ & 0.0009122 & -0.003571 & 0.001818 & 0.002099 & 0.4974 & 0.5091 \\
$a_{1}$ & $2.371 \mathrm{e}-4$ & $-2.52 \mathrm{e}-6$ & $9.080 \mathrm{e}-4$ & $9.257 \mathrm{e}-4$ & $4.863 \mathrm{e}-08$ & $-9.765 \mathrm{e}-08$ \\
$a_{2}$ & $1.717 \mathrm{e}-8$ & $6.145 \mathrm{e}-09$ & $6.657 \mathrm{e}-9$ & $1.164 \mathrm{e}-8$ & $1.539 \mathrm{e}-11$ & $1.049 \mathrm{e}-10$ \\
$a_{3}$ & $-2.412 \mathrm{e}-11$ & $-7.113 \mathrm{e}-12$ & $-7.225 \mathrm{e}-12$ & $-1.046 \mathrm{e}-11$ & $-1.166 \mathrm{e}-13$ & $-1.336 \mathrm{e}-13$ \\
$a_{4}$ & $1.836 \mathrm{e}-14$ & $4.531 \mathrm{e}-15$ & $3.239 \mathrm{e}-15$ & $4.057 \mathrm{e}-15$ & NA & NA \\
$a_{5}$ & $-7.88 \mathrm{e}-18$ & $-1.666 \mathrm{e}-18$ & $-5.07 \mathrm{e}-19$ & $-5.75 \mathrm{e}-19$ & NA & NA \\
$a_{6}$ & $1.891 \mathrm{e}-21$ & $3.523 \mathrm{e}-22$ & NA & NA & NA & NA \\
$a_{7}$ & $-2.351 \mathrm{e}-25$ & $-3.972 \mathrm{e}-26$ & NA & NA & NA & NA \\
$a_{8}$ & $1.163 \mathrm{e}-29$ & $1.847 \mathrm{e}-30$ & NA & NA & NA & NA
\end{tabular}

\section{References}

[1] C.J.Gardner, "Heavy Ion Parameters for 1997-1998, and some preliminary parameters for BAF “, AGS/AD Technical Note no.473, November 6, 1997.

[2] C.J.Gardner,W.vanAsselt,"Booster Tune Control Limits at High Field", Booster Technical Note No.220, February 10, 1993.

[3] K.A.Brown et al, "Resonant extraction parameters for the AGS Booster", 2001 U.S. Particle Accelerator Conference, Chicago, Illinois.

[4] E.Bleser, "Booster Short Quadrupole Production Measurements", Booster Technical Note No. 174, September 12, 1990.

[5] E.Bleser, "Booster Long Quadrupole Production Measurements", Booster Technical Note No. 176, September 13, 1990.

[6] R.Bianco and E.Bleser, "Summary of the Magnetic Measurements for the Short Booster Quadrupoles", Booster Technical Note No. 226, August 15, 1995.

[7] R.Thern, "Booster Dipole Production Measurements", Booster Technical Note No. 190, March 131991.

[8] C.J.Gardner, Private Communication; unpublished Booster Study.

[9] W.T.Weng, Department memo to D.Lowentstein. June 12, 1990. 